\title{
GESTÃO DO CONHECIMENTO EM SERVIÇOS DE TI: UM ESTUDO DO USO DO MODELO ITIL-SKMS EM MONITORAMENTO DE INFRA- ESTRUTURA DE TI
}

\section{KNOWLEDGE MANAGEMENT IN IT SERVICES: A STUDY OF THE USE OF ITIL-SKMS FRAMEWORK IN IT INFRASTRUCTURE MONITORING}

\author{
Gilmar Souza Santos ${ }^{1}$; Fernando Celso de Campos $^{2}$ \\ ${ }^{1}$ Universidade Metodista de Piracicaba - UNIMEP - PPGEP - Santa Bárbara d'Oeste - Brasil \\ gissantos@unimep.br \\ ${ }^{2}$ Universidade Metodista de Piracicaba - UNIMEP - PPGEP - Santa Bárbara d'Oeste - Brasil \\ fccampos@unimep.br
}

\begin{abstract}
Resumo
A gestão de conhecimento em serviços de tecnologia da informação (TI) tem assumido importância cada vez maior, diante dos desafios de um mercado cada vez mais globalizado. Existem provedores de TI no mercado mundial que estão investindo fortemente em gestão de conhecimento e inovação há algum tempo, a exemplo das empresas indianas. Neste contexto, o objetivo deste artigo é apresentar uma aplicação de um modelo de gestão do conhecimento em serviços de TI. Parte-se de um modelo teórico, o SKMS (Service Knowledge Management System), apresentado na versão do ITIL v3 - biblioteca de melhores práticas em gestão de serviços de TI. A seguir, com base em uma pesquisa-ação, estuda-se a aplicação do modelo dentro de um Global Operating Center ou Centro Global de Monitoramento de Infra-Estrutura de TI. Busca-se, finalmente, resultados significativos na criação, armazenamento e recuperação, assim como na integração do modelo à gestão do conhecimento do provedor. Apresenta-se, como conclusão, a importância do modelo na formação de competências essenciais de provedores de TI.
\end{abstract}

Palavras-chave: Gestão do Conhecimento; SKMS; ITIL; Serviços de TI.

\section{Introdução}

O Brasil começa a se destacar como destino viável para serviços de Tecnologia da Informação (TI), alternativo a outros países como a Índia. Os clientes internacionais começam a enxergar as vantagens competitivas do Brasil em relação a outros países. Neste contexto, um elemento diferenciador será a gestão do conhecimento em serviços de TI. A disponibilidade e a qualidade da mão-de-obra, o conhecimento e a inovação são fatores essenciais na hora de decidir pela localização de um serviço de TI, uma vez que as tarefas transferidas em geral envolvem grande 
movimentação e complexidade como processamento de pedidos e monitoramento de rede. Um indicador importante neste contexto é o grau de gestão do conhecimento em serviços de TI.

O conhecimento se tornou um ativo muito importante em serviços de TI. Desta forma, esta pesquisa tem por objetivo apresentar uma aplicação de um modelo de gestão de conhecimento aplicado a serviços de infra-estrutura de TI. Demonstramos, por meio de uma pesquisa-ação, a busca, organização, recuperação e disseminação do conhecimento na área estudada. Este conhecimento em serviços de TI foi estruturado e automatizado para permitir a sua difusão e uso estratégico na área. Avaliou-se alguns modelos do mercado e o mais indicado foi o SKMS (Service Knowledge Management System). Este trabalho, realizado em paralelo a uma implantação da certificação ISO/IEC 20000 - norma relacionada às melhores práticas em gestão de serviços de TI -, foi desenvolvido dentro de um provedor, aplicando conceitos científicos.

\section{Metodologia de Pesquisa}

A metodologia utilizada teve caráter exploratório e de pesquisa-ação. $\mathrm{O}$ aspecto exploratório está relacionado ao levantamento bibliográfico, baseado principalmente no modelo SKMS do ITIL v3. O objetivo foi a investigação deste modelo e a formulação dos possíveis benefícios de sua adaptação a um serviço de infra-estrutura de TI. Conforme Thiollent (2005), a pesquisa-ação é concebida e realizada em estreita associação com a resolução de um problema, no qual os pesquisadores e os participantes da situação ou do problema estão envolvidos de modo cooperativo ou participativo. Seguindo este padrão, esta pesquisa teve como base uma participação na concepção e implantação do modelo SKMS dentro do NOC de um grande provedor nacional de TI, com operações na America Latina, EUA, Europa e Ásia, 14 centros de desenvolvimento, full it service provider, 200 clientes, 85 centros de atendimentos técnicos, atuação em Application Outsourcing, IT Services, ERP Solutions, Fábrica de Software, Consultoria e Revenda de Equipamentos de TI. O provedor pesquisado possuía ainda 5.400 funcionários, sendo 200 em sua área interna de TI. Esta empresa utilizava ferramentas de gestão de conhecimento open source em sua área de Inovação \& Conhecimento, a exemplo do Open CMS e Afresco para Gestão de Conteúdo e Liferay como plataforma de Gestão de Conhecimento. As ferramentas eram totalmente aderentes às recomendações deste trabalho.

O objetivo da pesquisa era resolver um problema, com a máxima interatividade possível com a equipe local e auxiliar no objetivo principal que era a conquista da certificação ISO/IEC 20000, conforme os conceitos apresentados em Cauchick Miguel (2008). Uma meta atual do provedor é conseguir estar entre as 10 maiores empresas mundiais em TI, por meio de iniciativas como gestão do conhecimento e inovação, o que justificou e motivou o trabalho. 
Durante os trabalhos, outros modelos e práticas de gestão do conhecimento foram avaliados quanto à aderência em gestão de infra-estrutura de TI. Inicialmente avaliou-se os processos OID Organizational Innovation and Deployment do nível 5 do CMMI 1.2 Dev, Gestão de Conhecimento em Projetos do IPMA e também as Knowledge Management Practices do e-SCM/SP. O OID está fortemente vinculado a processos de software, o IPMA a gestão de projetos e o e-SCM está em um formato descritivo (alto nível) para gestão de outsourcing. Optou-se, desta forma, em pesquisar e implantar de forma mais detalhada o SKMS pela sua aplicação imediata em gestão de serviços de infra-estrutura de TI.

\section{Visão Geral - Gestão de Conhecimento}

Os dados podem ser considerados como sendo uma seqüência de números e palavras, sob nenhum contexto específico. Quando os dados são organizados com a devida contextualização, há a informação. Já o conhecimento é a informação organizada, com o entendimento de seu significado (SANTIAGO JR., 2004). Entre estes três elementos, os dados são aqueles que possuem menor valor. Normalmente, eles precisam ser manipulados e tratados para conterem algum valor e, a partir daí, se transformarem em informação. Os dados estão, normalmente, disponíveis no dia-a-dia. O grande desafio é a seleção daqueles que são importantes.

Conforme o ITIL v3 (2007) e mostrado na figura 01, uma gestão de conhecimento é normalmente representada através de um gráfico DIKW - Data, Information, Knowledge e Wisdom (Dados, Informações, Conhecimento e Sabedoria). O dado é uma série de fatos sobre eventos. Captura-se um volume de dados em banco de dados estruturados como sistemas de gestão de serviços de TI ou em uma base de dados de configurações (CMDB). A informação proporciona um contexto para os dados. Informação é normalmente arquivada em conteúdo semi-estruturado como documentos, e-mail e multimídia. O conteúdo deve ser fácil para capturar, consultar, encontrar, reusar e aprender com base nas experiências. O conhecimento é composto por experiência tácita, idéias, iniciativas, valor e comportamentos individuais. As pessoas conseguem conhecimento por conta própria ou da experiência de seus pares, assim como também a partir de análise de informações e dados. Sabedoria é o último discernimento do material e possui aplicação e conscientização contextual para proporcionar um forte senso comum de julgamento. 
Figura 01 - Modelo DIKW

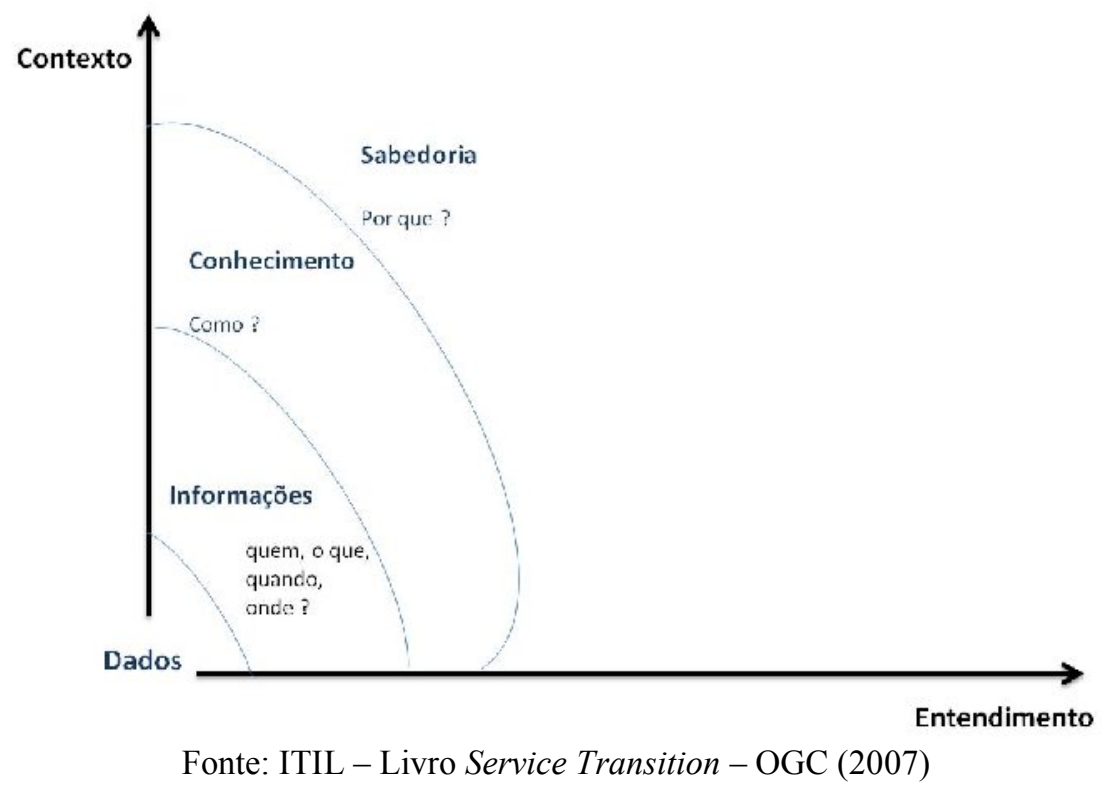

Segundo Santiago Jr (2004), a transformação da informação em conhecimento é possível a partir de:

- Comparação: entendimento sobre como as informações relativas a um determinado assunto podem ter alguma relação ou aplicação em outras situações;

- Conseqüência: implicação que determinada informação pode trazer para a tomada de alguma decisão e/ou ação;

- Conexão: relação entre a informação adquirida e um conhecimento já existente;

- Conversação: interpretação daquela informação a partir do entendimento sobre o que as pessoas pensam sobre ela.

A Gestão de Conhecimento cuida de agregar valor às informações filtrando, resumindo e sintetizando estas, e dessa forma, desenvolvendo um perfil de utilização pessoal que ajuda a levá-las à ação. Segundo Mergel et al. (2007) conhecimento implica em capacidades emocionais e cognitivas assim como habilidades relacionadas ao aspecto físico, que permitem tomar ações. Pode ser entendido como "saber como e por quê". Conhecimento é informação compreendida que habilita a ação. Conhecimento é a soma do que é percebido, descoberto, validado e aprendido (SABBAG, 2007). O gerenciamento do conhecimento, por sua vez, aplica as melhores práticas de atividades de gerenciamento para conhecimento dos importantes recursos da organização e dos clientes. Goodman e Colier (2007) sugere que o retorno sobre os serviços ofertados vem de uma efetiva gestão integrada do conhecimento, incluindo a Voz dos Empregados (VOE), Voz do Cliente 
(VOC), Voz do Negócio (VOB) e Voz do Processo (VOP). Mergel et al. (2007) acrescenta que o processo de gestão do conhecimento é composto por:

- Criação e aquisição do conhecimento;

- Organização e armazenamento do conhecimento;

- Integração e distribuição do conhecimento;

- Aplicação e adaptação do conhecimento;

- Avaliação e refinamento do conhecimento.

Conforme Takeuchi e Nonaka (2008), o conhecimento explícito, ou codificado, é o conhecimento cuja transmissão se dá através da linguagem formal e de forma sistemática. Pode ser armazenado e compartilhado, logo, mais fácil de gerenciar, podendo ser armazenado em normas, manuais e livros. $\mathrm{O}$ conhecimento não-explícito é igual ao conhecimento tácito. $\mathrm{O}$ conhecimento tácito é o conhecimento que a pessoa possui, incluindo suas habilidades. É pessoal, não pode ser expressado formalmente. É intrínseco à pessoa. Takeuchi e Nonaka (2008) destacam também que uma organização cria e utiliza conhecimento convertendo o conhecimento tácito em conhecimento explícito, e vice-versa. Para tornar possível a utilização dos conhecimentos da empresa é necessária a localização de suas fontes. Isto é feito através do mapeamento. Somente por meio dele é possível identificar os especialistas, pessoas com conhecimento de determinados assuntos, e localizar o acervo intelectual da empresa.

Segundo Santiago Jr (2004) muitas vezes, mais importante que importar experiências bemsucedidas é localizar as ilhas de deficiências internas e promover a disseminação das boas práticas. O mapeamento facilita a localização dos detentores de conhecimento, agilizando sua disseminação e a formação de equipes de trabalhos para novos projetos. Hansen (2009) acrescenta que existem três tipos de disseminação de conhecimento que podem ser utilizados em provedores de serviços: a) venda cruzada; b) transferência de melhores práticas e c) inovação a partir de combinação de tecnologias, serviços e processos. Existe, porém, a necessidade de uma análise do custo de oportunidade, custo da colaboração e retorno desta disseminação (HANSEN, 2009). No caso do centro de monitoramento de infra-estrutura de TI, o conhecimento passou pela análise de gap de competências. A partir de análise de perfis e experiências dos profissionais, foi fornecida uma ajuda para para a análise dos conhecimentos que precisavam para desenvolver suas atividades e ajudar na melhoria do conhecimento da área e da empresa como um todo. 


\section{Modelo SKMS - Sistema de Gestão do Conhecimento em Serviços de TI}

O Modelo SKMS representa as melhores práticas de gestão do conhecimento aplicadas à gestão de serviços de TI. Está incorporado na versão 3 do ITIL (IT Infrastructure Library). Consiste em quatro camadas, obedecendo a visão: dado, informação, conhecimento e sabedoria, conforme abaixo:

- Camada de Apresentação: nesta camada o objetivo é a colaboração e publicação das informações para utilização por todos os participantes da área. Busca-se o conhecimento e utiliza-o para resolver outros. Existem métricas para avaliar se o conhecimento está sendo bem aplicado. Nesta camada é realizado o planejamento de capacidade na visão do negócio, prevendo novos contratos para o provedor de TI. Realiza-se também a governança e elaboração de portfólio sobre todos os serviços de TI;

- Camada de Processamento de Conhecimento: realiza-se busca e análise dos dados através de portais de consulta. Estas informações podem estar online para acrescentar velocidade na tomada de decisão. Também é realizada modelagem de dados para uso em gestão de capacidade e análise de tendências;

- Camada de Integração de Informação: nesta camada, é mantido o sistema de gestão de conhecimento e de esquemas gerais. Os processos ITIL/ISO 20000 são relacionados para fornecerem informações. Realizam-se desenhos dos processos (SIPOC), como também catálogos de serviços e plano de capacidade técnico de TI;

- Dados e Informações: nesta camada os dados não estruturados são formatados e digitalizados. As base de dados de configurações (CMDB) e informações de sistemas como o ERP são montadas e executadas. 
Figura 2 - Modelo SKMS

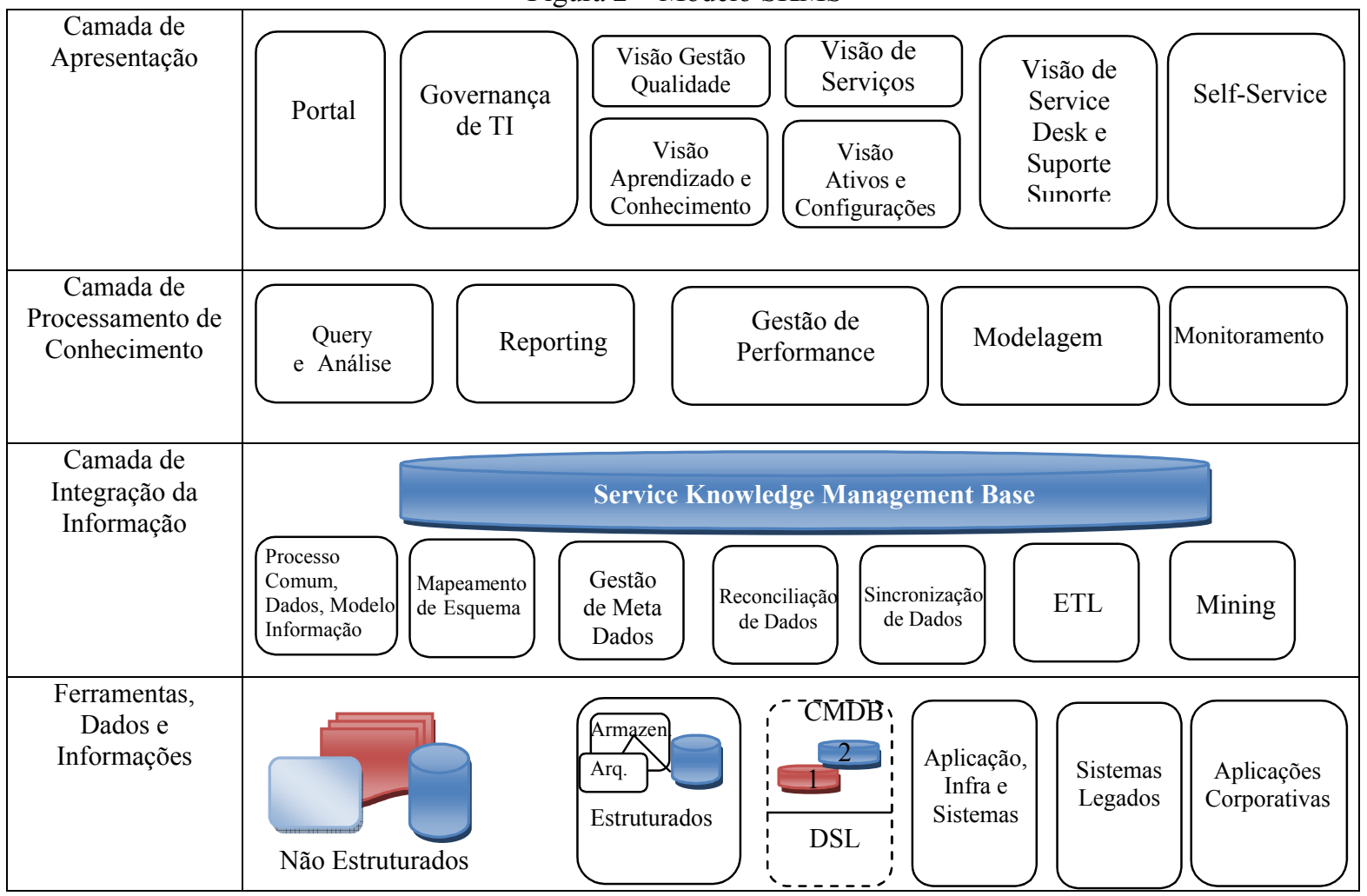

Fonte: traduzido e adaptado do Service Transition ITIL v3, OGC (2007)

O Modelo SKMS, mostrado na figura 2, é parte integrante da biblioteca ITIL v3, em seu livro TS- Transição de Serviços. O modelo considera, dentro da gestão de serviços de TI (GSTI), que um sistema de gestão de conhecimento é montado e armazenado dentro de uma base de dados, podendo ser recuperado e disseminado para toda a organização (ITIL ST, 2007). Dentro deste conhecimento leva-se em conta uma considerável quantidade de dados, os quais são suportados por um repositório lógico central ou Sistema de Gestão de Configuração (CMS) e a Base de Dados de Configuração (CMDB). O SKMS também abrange vários conceitos de base de conhecimento, incluindo:

- Experiência da equipe;

- Registros periféricos, ex. tempo, quantidade e ambiente de usuários, gráficos de performance da organização;

- Registros de configurações, capacidade, disponibilidade, segurança e outros;

- Requisitos de fornecedores e parceiros, capacidades e expectativas;

- Nível de habilidade de usuários;

- Conhecimento de elaboração de propostas de serviços de TI. 


\subsection{Estratégia de Gestão de Conhecimento}

Segundo o ITIL ST (2007), a estratégia para a gestão de conhecimento deve considerar um modelo de governança, papéis e responsabilidades nas mudanças organizacionais, políticas, processos, procedimentos, tecnologia, e indicadores de performance. Na gestão estratégica de um provedor de TI um dos desafios fundamentais é que, apesar do maior acesso a informações, paradoxalmente, existem dificuldades de gerar conhecimentos a partir destas informações. Isso porque está difícil distinguir sinais dos ruídos. Cabe a essas empresas identificarem as mudanças que acontecem no ambiente que irão afetá-las; discernirem quais conhecimentos emergentes são modismos e quais são verdadeiramente transformadores, e, por fim, entenderem quais iniciativas merecerão tempo, atenção e investimento da empresa (CHRISTENSEN, 1997). Neste aspecto, duas necessidades - negócio e compliance - representam a estratégia de gestão de conhecimento a ser atendida e que está representada nesta camada do modelo SKMS.

\subsection{Identificação, Captura e Manutenção do Conhecimento}

Envolve identificar o conhecimento que será útil, projetar um processo sistemático para organizar, analisar, armazenar e apresentar a informação de uma forma que melhore a compreensão das pessoas em uma área relevante. Também faz parte desta fase acumular conhecimentos ao longo dos processos e dos fluxos de trabalho, gerar novos conhecimentos, acessar conhecimento valiosos de fontes externas assim como capturar conhecimento externos e sua adaptação - dados, informações e conhecimento - de diversas fontes como banco de dados, websites, clientes, empregados, fornecedores e parceiros.

\subsection{Transferência de Conhecimento}

Conforme o ITIL ST (2007), durante o ciclo de vida dos serviços - estratégia, design, transição, operação e melhoria contínua - uma organização necessita focar em recuperar, compartilhar e utilizar seus conhecimentos obtidos a partir de soluções de problemas, aprendizado dinâmico, planejamento estratégico e tomada de decisões. Para atingir este objetivo, o conhecimento necessita ser transferido internamente na organização em pontos específícos do ciclo de vida. Um exemplo desta aplicação, citado pelo ITIL v3, refere-se aos scripts ou instruções de trabalho de atendimento de TI. Um dos objetivos é aumentar o suporte no $1^{\circ}$. nível de suporte, melhorando a satisfação do cliente e reduzindo custos com transferência de chamados. Já em projetos de serviços de TI, Ajmal e Koskinen (2008) relatam que as equipes são formadas de 
pessoas com diversas habilidades e conhecimentos trabalhando juntas em um período determinado. A transferência de conhecimentos para toda a organização é essencial. Há necessidade de aprender com a experiências e as práticas das atividades diárias. Outro aspecto da transferência de conhecimento é citado por Reich et al. (2008). Segundo os autores, uma gestão efetiva da transferência de conhecimento em projetos facilita a criação e integração do conhecimento, minimiza perda de conhecimento e preenche gaps da equipe ao longo da duração do projeto.

Para Sabbag (2009), disseminar conhecimento é muito mais do que divulgar. Consiste em

sair a campo para estimular a inovação, coletar idéias, realizar levantamentos, mapeamento e estudos, alimentando a base de conhecimentos. Além disto, deve-se reservar tempo e dedicação para ações de compartilhamento: eventos, reuniões, seminários e road-show.

\subsection{Gestão dos Dados e da Informação}

Segundo o ITIL v3 (2007), uma gestão dos dados e da informação deve ser realizado, a partir dos conhecimentos necessários para as decisões, disponibilidade dos dados, custo da captura, propriedade intelectual, políticas e padrões aplicáveis.

Os requisitos para a gestão dos dados e da informação incluem estabelecimento dos dados, itens da informação, conteúdo e forma (ex. técnicos, projetos, organizacional). São estabelecidos padrões comuns de conteúdo e formatos para facilitar o entendimento, gestão, requisitos de proteção, privacidade, segurança, propriedade, direitos de acesso, propriedade intelectual e patentes. Passa também pela definição de quem necessita acessar os dados e informações e quando deve acessá-los como também definição da importância do dado e da informação ao longo do tempo. Qualquer mudança realizada no processo de gestão de conhecimento deve passar pela gestão de mudanças de serviços de TI.

Deve-se criar e atualizar regularmente um modelo de informação de gestão de serviços que possibilite a criação, uso e compartilhamento da informação de forma flexível, rápida e econômica. Também deve ser definido um sistema que otimize o uso da informação e esquemas de dados, utilizados em toda a organização. Com os requisitos e arquitetura montados, a gestão de dados e informações para suportar a gestão de conhecimento é estabelecida. Esta fase envolve montagem dos dados e informações do ciclo de vida do serviço. Também são definidos os procedimentos, armazenamento, recuperação, autoridade, responsabilidade, direitos, obrigações, cópias de segurança e revisão de requisitos diante das mudança de tecnologia e de novas necessidades organizacionais. Nesta fase, uma medição para as transações do uso e utilização devem ser estabelecida e reportada. O controle de acesso aos dados como também procedimentos de workflow devem ser desenvolvidos e mantidos (ITIL SO, 2007). 


\subsection{Indicadores de Performance e Métricas de Gestão de Conhecimento em Serviços}

Para Sabbag (2009) qualquer aplicação de modelo de gestão de conhecimento, a exemplo do SKMS, deve agregar valor maior à organização. E esse valor deve ser tangível. È necessário medir os resultados, pelos seguintes motivos:

- É importante medir a efetividade da gestão do conhecimento;

- É necessário medir progressos no desenvolvimento da organização e de seu pessoal;

- É essencial avaliar intangíveis que geram valor à organização;

- É meio de fornecer informação relevante a acionistas, investidores e ao mercado

- É meio de refletir com maior propriedade o valor do negócio.

Desta forma, de acordo com o SKMS (ITIL ST, 2007), um business case é crítico para uma efetiva gestão dos conhecimentos em serviços de TI. Mostra o retorno financeiro e estratégico da iniciativa. Também é importante que as medições de sucesso sejam visíveis em todos os níveis envolvidos na implementação. As métricas que mostram o retorno, aplicáveis em um provedor de serviços de TI, incluem:

- Melhoria na experiência do cliente quanto à resolução rápida de uma consulta e solução (self-service) sem necessidade de acionar o suporte;

- Redução do tempo de transição do serviço;

- Alta percentagem de consultas e questões resolvidas via acesso a uma base de conhecimento;

- Maior acessibilidade e melhoria na gestão de padrões e políticas;

- Disseminação do conhecimento;

- Redução de tempo e esforço exigido para suportar e manter serviços;

- Redução do tempo para busca de informações para análise e solução de incidentes e problemas;

- Redução da dependência do conhecimento individual;

- Redução de incidentes e perda de produtividade categorizados como "ausência de conhecimento do usuário";

- Lições Aprendidas em Projetos;

- Quantidade de acesso ao SKMS. 
Em projetos, a exemplo da implantação e certificação da ISO 20000 em uma área de TI, um indicador muito importante são as lições aprendidas ao longo do projeto, retratado no modelo SKMS. Abdollahyan (2009) acrescenta que quando geramos e utilizamos lições aprendidas transformamos conhecimento tácito em explícito e o explícito em tácito, respectivamente. $\mathrm{O}$ autor cita três situações de boas práticas para obtenção de resultados em gestão do conhecimento por meio das lições aprendidas:

\section{- Transformação de conhecimento tácito em tácito e conhecimento tácito em explícito:}

ocorre em reuniões facilitadas pelo escritório de projetos ao final de cada fase do projeto, visando levantar, documentar e compartilhar as lições aprendidas;

- Transformação de conhecimento tácito em explícito: ocorre quando, a qualquer momento, membros da equipe e os demais stakeholders registram seus comentários, sugestões e críticas, que podem ser revistas nas reuniões de final de cada fase;

- Transformação de conhecimento explícito em explícito: quanto técnicos ou auditores da qualidade realizam suas tarefas técnicas e gerenciais - desenvolvimento de uma proposta ou solução, elaboração de uma EAP, revisão da qualidade, avaliação e verificação de escopo. Neste caso, a fonte do conhecimento é explícito tais como documentos desse mesmo projeto ou de outros projetos, evidências observadas e anotadas;

- Transformação de conhecimento explícito em tácito: quando esses mesmos técnicos consultam a base de dados de conhecimento para reutilizar as lições aprendidas nas mesmas tarefas mencionadas no item anterior.

\section{Implantação do Modelo em um centro de monitoramento de infra-estrutura de TI}

Um modelo baseado no SKMS foi implantado, de forma reduzida, em uma grande empresa de TI brasileira, particularmente dentro do seu NOC - Network Operation Center, como auxilio na busca da certificação ISO/IEC 20000 de melhores práticas em gestão de serviços de TI. O caso foi utilizado neste artigo em forma de pesquisa-ação. O objetivo foi contribuir com o projeto e com o conhecimento científico. O desenvolvimento e implementação do SKMS ajudou a reduzir o custo de manutenção e gerenciamento de serviços, por meio de aumento da eficiência e dos procedimentos de gestão operacional. Reduziu também os riscos de ausência de mecanismos apropriados. Partiu da premissa de que todo o conhecimento vinculado aos serviços de TI, prestados pela área, necessitava estar integrado e alinhado com a perspectiva do negócio. 
Figura 3 - SKMS Aplicado em um NOC

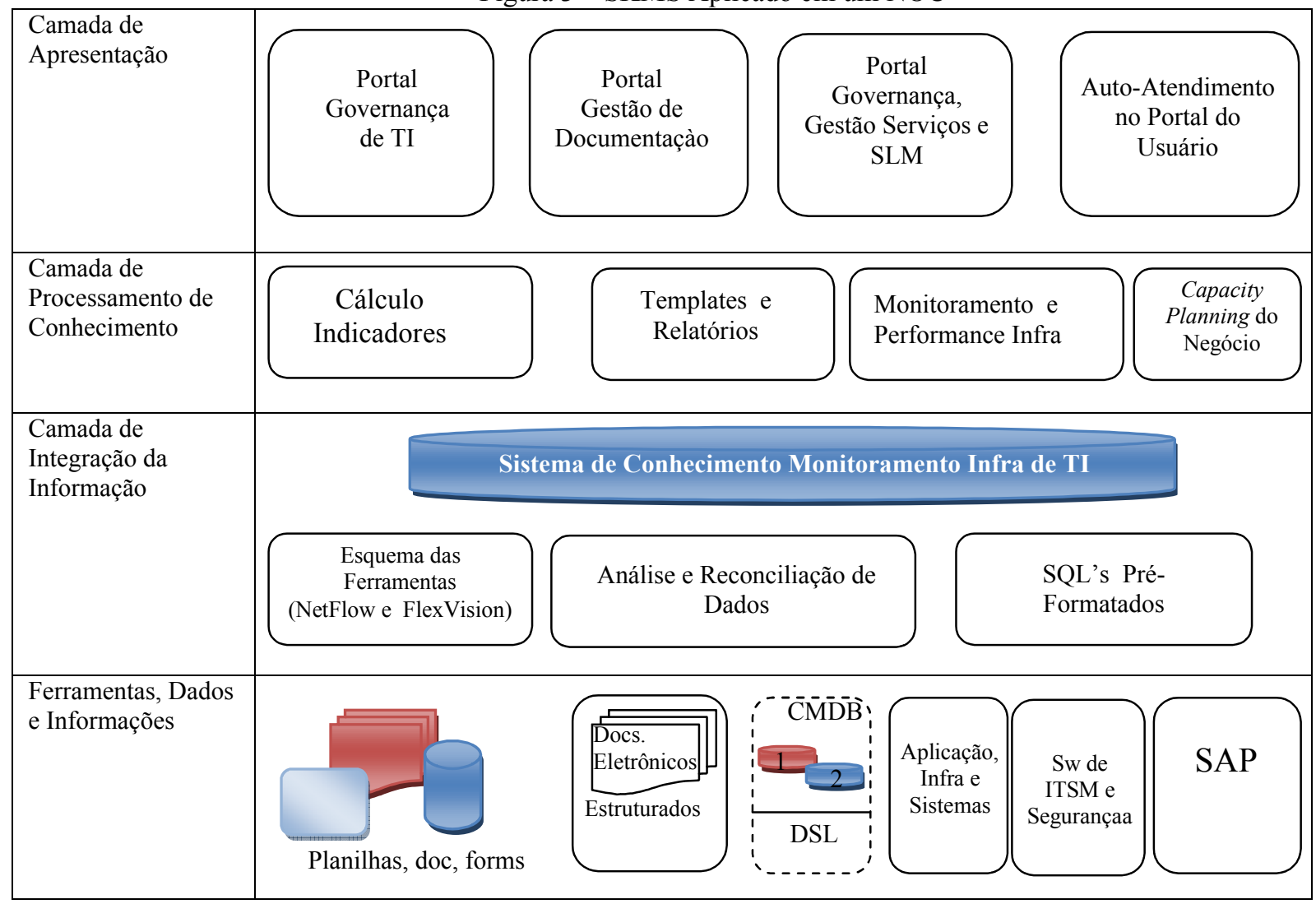

Fonte: autores (2008)

Conforme mostrado na figura 3, na camada de apresentação foi desenvolvido um portal de inovação e conhecimento para rede social interna, colaboração, coleta de idéias, propostas e inovações para melhorias dos serviços do NOC. Um dos objetivos foi mostrar que a partir de soluções originadas de colaboração e inovação, podem ser realizadas melhorias na infra-estrutura de TI. O portal de gestão de documentação possibilitou o armazenamento e recuperação da documentação do sistema de gestão de qualidade, no conceito de ECM (Enterprise Content Management). Segundo Parkinson e Baker (2005), informações de governança devem gerar suporte ao negócio, adequação às leis e regulamentos e também deve gerar resultados confiáveis e transparentes. Neste aspecto, o portal de governança possibilitou um front-end de apresentação de indicadores de processos e balanced scorecard. Ressalta-se que a solução implantada também poderia ter sido consolidada em um único portal (centralizador) através de recursos de web 2.0 como portlets, widgets e mashups.

$\mathrm{Na}$ camada de processamento de conhecimento, foi implantado um book de indicadores, baseado nos processos de serviços de monitoramento de infra-estrutura de TI como gerenciamento de eventos, incidentes, problemas e mudanças. Os templates e relatórios foram elaborados para suportar a necessidade de modelos e exemplos para os relatórios gerenciais e informações gerenciais em portais. Através do monitoramento e análise de performance, foram coletados elementos para o planejamento técnico de capacidade. A obtenção dos dados de planejamento de 
capacidade do negócio somente foi possível após a disponibilização das oportunidades de negócio do provedor de serviços (pipeline). Estabeleceu-se um modelo padrão para a cálculo de capacidade de inclusão de um novos serviço no NOC. O planejamento de capacidade foi possível a partir do cálculo dos recursos consumidos por cada unidade de serviço. A capacidade do negócio do provedor para seu serviço de infra-estrutura foi um dos elementos chaves para o SKMS.

Um fator importante no processo foi a disseminação dos benefícios da gestão de conhecimento em todas as áreas que faziam interface com a área escopo. Erros detectados em serviços durante a sua transição foram registrados e analisados. O conhecimento resultante ficou disponível para a operação dos serviços. O SKMS pressupõe que a equipe de gestão de problemas é a responsável em coletar o conhecimento, normalização dos dados, manutenção e disponiblizaçào dos scripts para o gerenciamento de incidentes.

$\mathrm{Na}$ camada de integração de informações a área desenvolveu consultas padrões para coleta de informações de análise de performance, capacidade e monitoramento. Também foram determinados padrões para as bases de dados das ferramentas de análise de rede, monitoramento e sistema de gestão de serviços, utilizando a linguagem SQL. A informação também necessitou ser armazenada na base de conhecimento de gestão de serviços (BDGS). Nesta base também foram armazenados todo o conhecimento obtido com a infra-estrutura de TI, reuso, esquemas de dados, padrões de consulta, capacidade, monitoramento, disponibilidade e performance. O repositório do sistema de conhecimento ficou concentrado nesta camada. Ele foi obtido a partir das consultas nos diversos dados disponíveis na camada inferior do modelo.

$\mathrm{Na}$ camada de avaliação e ferramentas, foi criada uma base de dados de configurações (CMDB) usando recursos já existentes na ferramenta de service desk.. Neste banco de dados, foram armazenados e relacionados todos os itens de configurações do centro de monitoramento a exemplo de contratos, propostas, planos de capacidade, ativos de rede, servidores, ferramenta de monitoramento, SLA, funções, documentação e outros. Todos foram relacionados, formando uma base de conhecimento para avaliação de impacto das mudanças realizadas como também como instrumento de análise de incidentes, problemas e tomada de decisões. Documentos e planilhas em papel, catálogo de serviços, planilhas de capacidade, disponibilidade, atas de reuniões, anotações e manuais foram digitalizados e preparados para inclusão nas ferramentas. Nesta camada foram implantadas as ferramentas de service desk e de monitoramento. Informações de orçamento e custos em serviços de TI foram obtidas a partir do sistema ERP para armazenamento posterior no sistema de gestão de conhecimento. 


\section{Recuperação do conhecimento explícito}

A implantação do SKMS consistiu na localização e no resgate do conhecimento que já existia e que era necessário para a implantação do modelo dentro do centro de monitoramento. Dados de níveis de serviços, capacidade, disponibilidade, eventos, itens de configuração e mudanças foram elementos fundamentais na busca da certificação ISO/IEC 20000 como também para a melhoria de qualidade dos serviços da área. Foi definido, inicialmente, que seria realizado o levantamento do conhecimento explícito através dos registros, procedimentos, manuais, indicadores e instruções disponíveis no centro de monitoramento. A empresa já possuía um sistema de gestão de qualidade baseado na ISO 9001:2000 e ele foi identificado como o ponto de partida para este levantamento. Esta etapa foi a mais trabalhosa da implantação do sistema de conhecimento, por alguns motivos a exemplo de:

- Os documentos armazenados no sistema de qualidade estavam no padrão adequado, porém com conteúdo insuficiente para a norma ISO 20000;

- Existia também uma dificuldade no acesso aos documentos;

- Alguns dados estavam de forma totalmente não padronizadas como planilhas de capacidade, disponibilidade, testes de continuidade e riscos de serviços;

- Dados de competências, responsabilidades e perfis dos profissionais da área não estavam alinhados com o plano de treinamento da empresa, como também com as diretrizes da área de Recursos Humanos.

Ocorreu a necessidade de desenvolver padrões de perfis como também mapeamento de gaps de competências e conhecimentos - incluindo necessidades de treinamentos - adequados à realidade da área.

Após a recuperação, padronização e armazenamento do conhecimento explícito foi necessário alocar um responsável para efetuar a gestão do conhecimento. A sua função foi reunir e gerenciar o conhecimento do NOC como também integrá-lo ao modelo de gestão de conhecimento da empresa. Outro aspecto importante na recuperação da transformação do conhecimento tácito em explítico foi a formalização de lições aprendidas dos novos projetos implantados no NOC. Conforme Heldman (2005), as lições aprendidas são as informações coletadas e documentadas ao longo de um projeto que podem ser utilizadas para beneficiar o projeto atual, projetos futuros ou outros projetos que estejam sendo executados pela organização. Essas lições podem ser positivas ou negativas. Durante um projeto, conhecimento deve ser transferido, integrado, criado e explorado para criar novo valor organizacional (REICH, 2008). Desta forma, na certificação ISO/IEC 20000 a 
coleta e reuniões das lições aprendidas foi realizada durante todo as fases dos projetos de novos serviços na área escopo, incluindo reuniões, status report, issues, change request, indicadores, auditoria da qualidade, auditoria do escritório de projetos, alimentação e reuso da base de conhecimento. Assim, conseguiu-se contemplar na implantação do modelo todas as fases de recuperação e transformação do conhecimento citadas por Abdollanhyan (2009) e descritas no referencial teórico deste trabalho.

\subsection{Estrutura do Modelo de Gestão do Conhecimento}

Um padrão foi necessário para estruturar o conhecimento obtido para formação do sistema de conhecimento da área. A norma ISO/IEC 20000, que estava sendo implantada na área, serviu como direcionador para a classificação, facilitando a taxonomia. A montagem da estrutura do CMDB (Base de Dados de Configurações) utilizou determinações da norma, como vincular todos os itens de configurações aos serviços da área. Estes serviços e suas características foram obtidos a partir de um catálogo de serviços. Em resumo, a estrutura determinou que todo o conhecimento da área fosse canalizado para a prestação dos serviços aos clientes. O objetivo foi organizar o conhecimento necessário para o negócio da área. Esta indexação foi importante, por exemplo, na montagem de um plano de capacidade. Criou-se condições para calcular qual o conhecimento necessário para cada unidade de serviço (ex. serviço de gerenciamento de eventos).

\subsection{Tecnologia utilizada na implementação do modelo}

Após a definição de como seria organizado o sistema de conhecimento, iniciou-se uma modelagem, com o objetivo de montar um sistema para armazenamento, recuperação e difusão do conhecimento. O objetivo era atender as camadas de processamento (registro e atualização) e apresentação da informação (consultas com objetivos de tomada de decisões). Neste momento também foram definidos padrões da camada de apresentação como browsers, portlets, widgets, mashups, content management e wikis para a distribuição e colaboração das informações.

\subsection{Resultados Obtidos}

A implantação do sistema de conhecimento em serviços de TI, baseado no SKMS, permitiu ao NOC obter de forma consistente a certificação ISO/IEC 20000 em julho/2008. A obtenção desta certificação foi uma clara sinalização para o mercado do uso de melhores práticas para gestão de serviços de TI. Como segundo resultado mais importante, o sistema montado possibilitou a 
utilização adequada dos conhecimentos e das experiências existentes dentro do centro de monitoramento para a absorção de novos clientes e de novos serviços de TI. Finalmente, percebeuse uma grande melhoria do relacionamento com o cliente e com o negócio do provedor de TI. Os portais de documentação, permitiram armazenar documentações e práticas necessárias para a ampliação da certificação para as demais áreas do provedor, bem como permitiu uma combinação do conhecimento adquirido no projeto com outras práticas utilizadas. Uma análise do resultado final está demonstrada no quadro 1.

Quadro 1 - Análise dos Resultados da Implantação do Modelo SKMS

\begin{tabular}{|c|c|}
\hline Qual a Visão ? & Implantar o modelo SKMS dentro do projeto ISO $20000 \mathrm{em}$ um provedor de TI \\
\hline Onde Estava? & $\begin{array}{l}\text { Estruturação de conhecimentos tácitos e explícitos, documentos com conteúdo } \\
\text { insuficiente, dificuldade de acesso às informações de documentos e práticas, } \\
\text { necessidade de integração de dados e informações com demais áreas envolvidas } \\
\text { no projeto, padronização de dados não estruturados, necessidade de integração } \\
\text { com banco de dados de configurações, necessidade de modelo para } \\
\text { armazenamento das documentações dos processos da ISO 20000, necessidade de } \\
\text { integração com o negócio. }\end{array}$ \\
\hline Qual o resultado almejado? & $\begin{array}{l}\text { Modelo SKMS adaptado e implantado dentro do projeto ISO/IEC } 20000, \\
\text { conforme as melhores práticas do ITIL }\end{array}$ \\
\hline $\begin{array}{l}\text { O que foi feito para chegar até o } \\
\text { resultado? }\end{array}$ & Projeto de implantação do modelo SKMS integrado à ISO/IEC 20000 \\
\hline $\begin{array}{l}\text { Como a empresa ficou sabendo } \\
\text { que atingiu o resultado? }\end{array}$ & $\begin{array}{l}\text { Modelo SKMS implantado, certificação ISO/IEC } 20000 \text { obtida e comparação } \\
\text { dos indicadores abaixo com os medidos antes da implantação da norma: } \\
\text { - } \quad \text { Produtividade da equipe } \\
\text { - } \quad \text { Resolução de problemas com uso do KB } \\
\text { - } \quad \text { Reuso de documentação } \\
\text { - } \quad \text { \% utilização de práticas do ITIL e ISO } 20000 \\
\text { - } \quad \text { Quantidade mensal de novos scripts de atendimentos } \\
-\quad \text { Qtde. capacitação mensais em melhores práticas } \\
-\quad \text { \% Uso de templates e relatórios padrões } \\
\text { - } \quad \text { \% Problemas resolvidos no próprio NOC } \\
\text { - } \quad \text { \% Aderência e Integração KB NOC com o KB da Empresa }\end{array}$ \\
\hline
\end{tabular}

Fonte: autores (2008)

No final dos trabalhos a expectativa era de aumentar os benefícios do SKMS, integrando este modelo com outra práticas e bases de conhecimento da empresa, a exemplo de Recursos Humanos, ERP (Enterprise Resource Planning), Engenharia de Software e também Consultoria.

\section{Conclusão}

O foco da pesquisa foi o estudo e aplicação do modelo ITIL-SKMS da biblioteca ITIL v3 em um centro de monitoramento de infra-estrutura de TI (NOC). O objetivo maior foi demonstrar que este modelo de gestão de conhecimento, alinhado com a tecnologia necessária, transforma-se em uma competência essencial em empresas de TI, na busca de vantagem competitiva no mercado de tecnologia da Informação. O artigo procurou também demonstrar que a implantação de um 
modelo de conhecimento em serviços de TI possibilita a utilização adequada dos conhecimentos e das experiências existentes em provedores. Esta ação facilita o desenvolvimento de novas ofertas ao mercado e de novos projetos. O trabalho enfatizou a necessidade de provedores de TI de gerenciar melhor os seus conhecimentos em serviços de TI. O conhecimento, obtido e administrado, pode ser utilizado como uma competência essencial para os provedores, agregando valor aos seus serviços e produtos de TI.

Este trabalho também procurou demonstrar para os provedores de TI a importância da adequada utilização da estruturação e consolidação dos dados e das informações existentes. Esta ação é uma grande facilitadora na montagem da gestão de conhecimento no serviço de TI. Este trabalho também procurou demonstrar para os provedores de TI a importância da adequada utilização da estruturação e consolidação dos dados e das informações existentes. Esta ação é uma grande facilitadora na montagem da gestão de conhecimento no serviço de TI. Como continuidade deste trabalho, recomenda-se uma pesquisa sobre os principais modelos e práticas de Gestão de Conhecimento em Tecnologia da Informação incorporados em metodologias como CMMI, PCMM, IPMA, e-SCM-SP e PMI, apontando um modelo prescritivo para aplicação em todas ás áreas de conhecimento de TI.

\begin{abstract}
IT Services Knowledge Management has assumed increasing importance, given the challenges of a market that is increasingly globalized. There are Global IT providers that are investing heavily in knowledge management and innovation for the longest time, like the Indian companies. In this context, the objective of this paper is to present an application of a knowledge management model in IT services. It starts with a theoretical model, the SKMS (Service Knowledge Management System), presented the version of the ITIL v3 library - best practice in IT Service Management. Then, based on an action-research studies model's application within a Network Operating Center. Finally, the paper presents significant results in the creation, storage and retrieval, as well integration of the model within the provider. Is presented in conclusion the importance of the model for provider's core competences.
\end{abstract}

Key-words: knowledge management; skms; itil; it services

\title{
Referências
}

ABDOllahyan, F. Gestão do Conhecimento em Projetos: O especialista responde. Mundo Project Management. v. 5, n. 27., p. 30-32, 2009.

AJMAL, M. M.; KOSKINEN, K.. U. Knowledge Transfer in Project-Based Organizations: An Organizational Culture Perspective. Project Management Journal. WILEY/PMI. V.39, N.1. , p. 7-15, 2008.

CAUCHICK MIGUEL, P. A.. Implementação do QFD para o Desenvolvimento de Novos Produtos. Capítulo 1 - A Abordagem da Pesquisa-Ação. 1a. ed. São Paulo: Atlas, 2008.

CHRISTENSEN, C.. The Innovator's Dilemma. 1a. ed. New York: Harper, 1997

GOODMAN, J.; COLLIER, C.D. Deliver Great Service by Listening and Adapting. Quality Progress. ASQ American Society for Quality. March, 2007.

HALVEY, J. K.; MELBY, B. M. Information Technology Outsourcing Transactions - Process, Strategies and 
Contracts. 2a. ed. New Jersey: 2005.

HANSEN, M.T. When Internal Collaboration Is Bad for Your Company . Harvard Business Review. V.87, n. 4, p. 62-69, 2009.

HELDMAN, K. Project Management. 3a. ed. New Jersey: Wiley Publishing, 2005

ITIL ST. ITIL - Service Transition Book - SKMS. Londres: OGC. 2007

ITIL SO. ITIL - Service Operation Book - SKMS. Londres: OGC. 2007

ITIL v3. Introduction to the ITIL Service Lifecycle. 1a. ed. Londres: OGC, 2007

MERGEL, I.; VONKORTZFLEISCH, H. F.O; PROLL, C.. Potentials of Social Networks for Knowledge Management with regard to the Development of Stable Competences and Dynamic Capabilities - Conceptualization and Case Study Results. ACM. Hawaii International Conference on System Sciences, 2007.

PARKINSON, M. J. A.; BAKER, N. J. IT and Enterprise Governance. Information Systems Control Journal. ISACA/ITGI. p. 10-14. V.3, 2005.

REICH, B. H.; GEMINO, A.; SAUER, C. Modeling the Knowledge Perspective of IT Projects. Project Management Journal. WILEY/PMI. V.39, p. S4-s14, 2008.

SABBAG, P. Y. Gerir projetos requer gerir conhecimentos. Mundo Project Management. v. 5, n. 27., p. 8-15. 2009.

SABBAG, P. Y. Espirais do Conhecimento - Ativando Indivíduos, Grupos e Organizações. $1^{\mathrm{a}}$. Ed. São Paulo: Saraiva, 2007.

SANTiAgo JR., J. R. S.. Gestão do Conhecimento - A Chave para o Sucesso Empresarial. 1ª. ed. São Paulo: Novatec, 2004.

SANTOS, G. S. Uma Proposta de Integração de CMDB Para Alinhamento de Processos de TI. XV CNASI Congresso Nacional de Segurança, Auditoria de Sistemas e Governança de TI. São Paulo: ISACA/CNASI, 2006

TAKEUCHI, H.; NONAKA, I. Gestão do Conhecimento. 1' ${ }^{\text {a }}$ ed. Porto Alegre: Bookman, 2008.

THIOllent, M. Metodologia da Pesquisa Ação. 16 ed. São Paulo: Cortez Editora, 2005.

\section{Dados dos autores:}

Nome completo: Gilmar Souza Santos

Filiação institucional: UNIMEP - Universidade Metodista de Piracicaba

Departamento: FEAU - PPGEP - Programa de Pós-Graduação em Engenharia de Produção

Função ou cargo ocupado: Doutorando em Engenharia de Produção

Endereço completo para correspondência (bairro, cidade, estado, país e CEP): Rua Barata Ribeiro, 156 - Ap 96 - 01308000 - Bela Vista - São Paulo-SP

Telefones para contato: $119113-9620114196-9349$

e-mail:gissantos@unimep.br 
Nome completo: Fernando Celso de Campos

Filiação institucional: UNIMEP - Universidade Metodista de Piracicaba

Departamento: FEAU - PPGEP - Programa de Pós-Graduação em Engenharia de Produção

Função ou cargo ocupado: Professor Titular

Endereço completo para correspondência (bairro, cidade, estado, país e CEP): Universidade

Metodista de Piracicaba - UNIMEP Programa de Pós-Graduação em Engenharia de Produção

Rodovia Santa Bárbara D’Oeste - Iracemápolis, Km.1 13450-000 - Santa Bárbara D’Oeste, SP

Telefones para contato: 19 3124-1767

e-mail:fccampos@unimep.br

Recebido para publicação em: 23/09/2009

Aceito para publicação em: 05/11/2009 